\title{
A Standardized SOA for Clinical Data Sharing to Support Acute
}

\section{Care, Telemedicine and Clinical Trials}

\author{
Roberta Gazzarata ${ }^{1,2}$, Mauro Giacomini ${ }^{1,2}$ \\ ${ }^{1}$ Department of Computer Science, Bioengineering, Robotics and Systems Engineering (DIBRIS), University of Genoa, Genoa, Italy \\ ${ }^{2}$ Healthropy, Savona, Italy
}

\begin{abstract}
Background: Developed countries are planning the creation of national EHR (Electronic Health Record) systems to modernize the healthcare field and improve its quality, security and efficiency.

Objectives: To support clinical data sharing, it is important that an EHR is designed to be integrated within an appropriate architectural context aimed to satisfy the needs of all actors involved in this information management by adding and integrating new functionalities to existing solutions.

Methods: SOA (Service Oriented Architecture) provides a good approach to promote the easy integration and alignment of a new and existing solution into a cohesive architecture. The HSSP (Healthcare Service Specification Program) was formed to adopt the SOA approach to guarantee interoperability between applications and distributed and heterogeneous devices, by providing a set of standards to design and develop specific services.
\end{abstract}

Results: The authors present a landscape architecture to support the collaboration between actors involved in the treatment of chronic diseases. The core of this architecture consists of services compliant to HSSP standards. Among these, the authors developed: Health Record Management Services, Health Terminology Services and Health Identity Services. The proposed architecture and these services have already been adopted in different systems: a telemonitoring system to support the continuity of care of CHF (Congestive Heart Failure) patients, two systems to share clinical data to manage clinical trials in both infectivology and ophthalmology.

Conclusions: The main advantage of the proposed architecture is its flexibility that allows it to be adapted over time and to be adopted in all health care scenarios.

\section{Keywords}

Electronic Health Record; Service Oriented Architecture; Clinical Data Sharing; Healthcare Specification Project

\section{Correspondence to:}

\section{Roberta Gazzarata}

Department of Computer Science, Bioengineering, Robotics and Systems Engineering (DIBRIS), University of Genoa Address: Via Opera Pia 13, 16145 Genoa, Italy E-mail: Roberta.gazzarata@dibris.unige.it
EJBI 2016; 12(1):en49-en57

received: April 19, 2016

accepted: April 29, 2016

published: May 20, 2016

\section{Introduction}

Technological-scientific progress in the medical field is extending the population's life expectancy with the consequence that a person can undergo many healthcare encounters in her/his lifetime. In order to correctly and efficiently treat a patient, it is essential that all medical staff collaborate and have a complete knowledge of his/her past experience and clinical history, particularly in the case of chronic diseases or acute events where the patient may be aided by a large number of clinicians and specialists.

In this complex scenario, the Electronic Health Record (EHR) represents the most suitable solution designed exactly to support these needs. The EHR is a digital repository for healthcare information related to a person's life- time with the goal of supporting treatment continuity, education and research, whilst always guaranteeing privacy protection [1. In many developed countries such as Italy [2], Austria [3], Luxembourg 4], Denmark [5], Norway 6], and the United Kingdom [7, the Healthcare Ministries are increasingly interested in the potential benefits provided by the EHR to modernize the healthcare field and improve its quality, security and efficiency, so consequently, they are planning the creation of national EHR systems [8, 9, 10].

Different steps of clinical data integration within the EHR are described in literature. Waegemann indicated the Automated Medical Record (AMR), the Computerized Medical Record (CMR) and the Electronic Medical Record (EMR) as different stages of automation within 
one health institution. The Electronic Patient Record (EPR) was also mentioned, whose purpose is to extend record integration across the borders of institutions. The successive step is the EHR which allows each responsible citizen to manage and have control over his/her own medical data [11, 12.

Even though Wargemann pointed out these phases of patient clinical data integration in 1999, at present there is not a common and effective automatic communication between all these entities. The implemented EHR solutions provide the citizen with a tool to insert and save his/her own clinical data in a manual way; in fact, the possibility to automatically feed information into the EHR is often missing, even in the easiest of cases, where automated instruments produce the information directly.

These solutions show other critical problems. Firstly, the formats typically used for information filing are not structured documents and simply represent digital copies of paper documents, such as pdfs, jpegs etc. [1. This lack of semantic management and organization of the information content does not allow the EHR to be automatically and easily accessible and interpretable when required. This is exactly the case of patients suffering from chronic and/or infectious diseases. For correct and efficient treatment, it is extremely useful to know the complete patient clinical history contained in the EHR, particularly when the health state of the patient becomes critical, in order to accelerate the emergency procedures, raising the chances of saving lives. In addition, all the data, collected during a patient's lifetime, would be highly useful, if made available to advanced research centers. In fact, the medical field is undergoing a significant change in the way healthcare professionals interact with patients' data. Clinical data is more and more often defined as valuable in helping to make decisions about patients' treatment [13, 14.

In order to support clinical data sharing, it is important that an EHR system is designed to be integrated within an appropriate architectural context aimed to satisfy the needs of all actors involved in this information management. In the UK, the National Health Service (NHS) invested in the largest civil IT project in the world, the National Programme for IT (NPfIT), with the aim of seeking to revolutionize the way care is delivered, improve quality and use NHS resources more effectively. Despite these high expectations, the NHS has historically experienced some high profile IT failures and the sponsors of the programme admitted that there remains a number of critical barriers to the implementation of the project [15]. Clinicians' reluctance to accept new IT systems at a local level is seen to be a major factor in this respect. In particular, findings show that clinicians often perceived that the IT systems, proposed by the NPfIT, would have little positive impact on making their job easier or improving patient care; although it was mentioned that there was no resistance to new technology as many new medical technologies had already been embraced [15]. Therefore the clinician's approval is fundamental for the effective suc- cess of every e-health application, which occurs when new technologies are designed with an effective collaboration between the physician and IT staff.

Another important aspect to be considered is that IT efforts to evolve are hampered by the extensive existing investments in hardware, software, and medical devices, which must continue to be supported by healthcare organizations, while being under increasing pressure to modernize systems. The Service Oriented Architecture (SOA) provides a highly feasible approach to promote the easy integration and alignment of a new and existing solution into a cohesive architecture [16.

Firstly, this paper describes the landscape architecture that the authors designed and proposed to allow the complete and effective collaboration between all the actors which can be involved in an overall care cycle, in order to manage both acute events and chronic illness. Then it presents the state of the implementation of this architectural solution which is being developed and employed in different systems to support the treatment of different diseases and the continuity of care. In particular, this architecture is applied to the management of certain chronic illnesses such as in the treatment of cardiovascular diseases, infectious diseases and eye infections and also to the surveillance of multi-resistant microorganisms.

\section{Methods}

The clinical data that is managed within the proposed solution depends on the particular class of patients considered by each implemented solution. For patients suffering from cardiovascular diseases the data is related to the complete clinical history and also to vital signs, if continuous monitoring is necessary after hospitalization, during the rehabilitation.

If the patients are affected by infectious diseases, for example, related to Human Immunodeficiency Virus (HIV) and to Hepatitis B/C Virus (HBV/HCV) the clinical data refers to specific blood tests indicated to monitor their health status.

If the patients are affected by degenerative eye diseases, the managed data is related to information collected during specific encounters (like the status of the vision and the objective description of the retina situation).

In the case of surveillance of multi-resistance to antibiotics the exchanged data are more varied, and consist of anamnestic information on the patient, location of where the infection was acquired, identification of the microorganism and its antibiogram spectra.

The actors who are involved in the treatment of these patients and diseases are:

- Departments and care units of Ligurian hospitals: Infectious Diseases Departments, Departments of Neurosciences, Ophthalmology and Genetics, Coronary Care Units

- General practitioners 
- Specialists

- Internal and external hospital laboratories

- The Ligurian regional EHR, also called "Conto Corrente Salute" ("Health Checking Account")

- Clinics for rehabilitation or the patient's home

- Advanced Ligurian and Italian research centers

- External Clinical Decision Support (CDS) systems (e.g. openCDS community [17])

In order to share the clinical data mentioned, two HL7 products, derived from the HL7 version 3 (v3) Reference Information Model (RIM) were used: the HL7 v3 Clinical Document Architecture Release 2 (CDA R2) and the HL7 v3 Virtual Medical Record (vMR). In particular, on one hand the authors adopted the CDA R2 [18] for the information transmission between hospitals, general practitioners, specialists, laboratories, the regional EHR, clinics or the patient's home and advanced research centers; on the other hand, the vMR data model was chosen to provide clinical information to external CDS systems [19.

The structure of CDA R2 is extremely generic and flexible, and is therefore adaptable to satisfy the requirements of different interoperability scenarios. For this reason, an Implementation Guide (IG), which constrains the CDA R2 specification, must be provided for each use case. The IG is usually produced by HL7 International, then each country-specific HL7 Affiliate organization is authorized to edit a national version appropriate for the local healthcare context. The choice of IG is related to the clinical and administrative data that are managed, which in turn depends on the particular class of patients considered by each implemented solution. At present, the HL7 Italian affiliate has not yet produced an Italian CDA R2 IG which can be adopted for the management of patients suffering from cardiovascular diseases, but it developed the Italian localization of the "Implementation Guide: CDA Release 2 - Care Record Summary Release 2 Discharge Summary" 20]. The authors decided to take into account this IG and if necessary a few CDS R2 sections from the "Implementation Guide: CDA Release 2 - Continuity of Care Document (CCD)" 21]. For the management of patients who are affected by infectious diseases the authors considered the HL7 Italy IG for Laboratory Reports [22, which represents the Italian localization of the IHE (Integrating the Healthcare Enterprise) Laboratory Technical Framework 23. Finally, for the management of patients affected by degenerative eye diseases, the Italian localization of the "Implementation Guide: CDA Release 2 - Care Record Summary Release 2 Discharge Summary" 20].

The SOA approach was utilized as a vehicle to transmit the clinical information across these health organizations. One of the SOA key principles lies in the ability to adapt the architecture over time, adding new services, replacing existing services and reconfiguring, all with minimal impacts to service consumers. The SOA reduces the amount of client point to point interfaces needed within a given environment 24 .

To design the reference architecture the authors took into account the specifications provided by the Healthcare Services Specification Project (HSSP). The HSSP is a program jointly promoted by the HL7 International and the Object Management Group (OMG) and is regulated by the Statement of Understanding (SOU) between HL7 International and the OMG. The HSSP was formed in 2005 in order to define health industry SOA standards that promote interoperability. In particular, the main HSSP objective is to use the SOA approach to provide and guarantee an effective interoperability between applications, and distributed and heterogeneous devices, which belong to independent socio-health system organizations. The aim of every HSSP project is the standardization of a specific service, which is related to a functional sociohealth domain, as a generic service. The intention is to standardize generic functions and protocols, which allow application and technical communication, in order to invoke, accept or reject and report the performance of these functions. The HSSP characterized the SOA services into three clear categories which are:

- Healthcare-Unique Services. This category calls-out service capabilities that are either unique to healthcare, or for which healthcare has unique requirements. For instance, both record management, clinical decision support and order management appear here.

- Business Services. Business-services describe those capabilities that support business competences or processes. Some examples are terminology, payroll, accounting, human resource management and demographics.

- Technical/Infrastructure Services. These services involve capabilities like service instance location, protocol/message routing, etc. [25].

This work focuses on the first two service categories mentioned above, which are the most interesting ones from a research point of view, as Technical/Infrastructure Services are mature components of the SOA, widely used in well-assessed distributed environments such as banking and assurance systems. The following objects from Healthcare-Unique Services and Business Services were selected in the present architecture:

- Health Record Management Services (HRMS) and Health Decision Support Services (HDSS) from the Healthcare-Unique Services category

- Health Terminology Services (HTS) and Health Identity Services (HIS) from the Business Services category.

HRMS are standardized services to manage patients' profiles and clinical history and the interfaces are defined by the Retrieve, Locate and Update Services (RLUS) Release 1 standard [26]. The RLUS standard provides a set 
of interfaces through which information systems can access and manage information within and between healthcare organizations. RLUS allows health data to be located, accessed and updated regardless of underlying data structures, security concerns or delivery mechanisms. It is independent of but compatible with underlying structures, including local security implementations, data models, or delivery mechanisms. By separating and exposing those aspects of resources that facilitate inter-organization workflows in a service layer, this specification abstracts the problem of interoperability away from underlying systems. It is not intended to replace existing systems or implementations, but to create an interface standard for a service-oriented layer to expose those healthcare assets and resources within an organization that are needed to meet business or medical needs.

The RLUS standard, as all HSSP products, is distributed through the HL7 Service Functional Model (SFM) which provides a service interface specification at a functional level (SFM for RLUS is available at [27]). An interface specification is defined by the Software Engineering

Institute (SEI) Software Architecture Glossary as a statement of what an architect chooses to make known about an element in order for other entities to interact or communicate with it 28. Starting from the HL7 SFM, the OMG develops the "Requests for Proposal" (RFP) which are the basis of the OMG standardization process. In this phase vendors and other submitters propose solutions which satisfy the requirements indicated in the RFP while leaving design flexibility to the submitters and implementation flexibility to the users of the standard. The result of this process is the OMG Service Technical Model (STM) which specifies the technical requirements of the service 29] (STM for RLUS is available at 30]).

HDSS are standardized services to research, query and execute modules to help in decision making and their interfaces are defined by Clinical Decision Support Services (CDSS) Release 1 standard 31. The CDSS standard provides interface specifications and technical requirements which are needed for a standardized approach for leveraging machine-executable medical knowledge in an application-independent manner. A Decision Support Services (DSS) receives patient data as the input and returns patient-specific conclusions as the output. In this way, it can significantly facilitate the implementation of systems that require patient-specific inference such as Clinical Decision Support (CDS) systems and quality reporting systems. CDS systems are solutions which provide physicians and other healthcare stakeholders with patientspecific assessments or recommendations in order to assist in clinical decision making. Examples of CDS systems include outpatient systems that attach care reminders to the charts of patients who need specific preventive care services, Computerized Provider Order Entry (CPOE) systems which provide patient-specific recommendations as part of the order entry process, and laboratory alerting systems which warn physicians when critical laboratory values are detected 31 .

HTS are standardized services to manage clinical and health codifications and terminologies and their interfaces are defined by Common Terminology Services Release 2 (CTS2) standard 29]. The CTS2 standard provides a consistent specification to develop service interfaces to manage, search and access terminology content, either locally, or across a federation of terminology service nodes, independent of the terminology content and underlying technological stack. Structured terminologies supply the basis for information interoperability by improving the effectiveness of information exchange within a specific domain. Specifically, the structured terminologies provide a tool to organize information and to define the information semantics using consistent and computable mechanisms. In a shared semantics environment, the CTS2 provides a modular and common set of behaviors which can be used to deal with a set of terminologies chosen by the clients. The service contributes to interoperability by supporting an easy access to the foundational elements of shared semantics [29, 32]. The HL7 SFM for CTS2 is available at 29] while the OMG STM is available at 32.

HIS are standardized services to define, update and generally manage identities and their interfaces are defined by Identification and Cross-Reference Service (IXS) Release 1 standard [33. The IXS standard provides a set of service interfaces to uniquely identify and index various kinds of entities (patients, providers, organizations, systems and devises) both within and across health organizations. The IXS allows any system which uses the service to maintain a common description for each entity and to manage the entities. The unique identifier and standard way to search, retrieve and manage entity data allows healthcare applications and healthcare enterprises to find, exchange and reference entity data while maintaining the data context and associations [33, 34. HL7 SFM for IXS is available at 33 . while OMG STM is available at 34 .

\section{Results}

\subsection{Proposed Architecture}

The landscape architecture, which the authors proposed to support the collaboration between actors involved in the treatment of chronic and/or infectious diseases and in the surveillance of antibiotic multi-resistant microorganisms, is represented in Figure 1. The core of this architecture consists of two Healthcare-Unique Services, the HRMS and the HDSS, and two Business Services, the HTS and the HIS.

The Health Record Management Service, whose interface is compliant to RLUS standards, permits the standardized transmission of clinical data within a clinical document between hospitals, general practitioners, specialists, laboratories, the regional EHR, clinics or a pa- 
tient's home and advanced research centers. The HRMS provides operations to allow an authorized client to get and put resources mapped using the HL7 v3 CDA R2. In particular, the put resource operations are used to share clinical data with other specific clients of the HRMS; typical examples are the cases in which:

- A department/care unit of a hospital or clinic/patient's home, a laboratory or a specialist wants to update the regional EHR

- A department/care unit of a hospital wants to contribute to medical trials by sending information to research centers.

When the HRMS receives a CDA R2 as a parameter of a put resource operation, it processes the header content to extract the author in order to know who is authorized, so as to re-address the document. Before sending this resource, the service has to modify some of the elements in its content. Indeed, in this CDA R2 it is possible that the code attribute used for the clinical statement (e.g. observation) belongs to a code system defined within the specific system (e.g. department/care unit or laboratory). This occurs in Italy, as the national effort to provide a standardized nomenclature was motivated by exclusively economic purposes, which are related to the refunds of outpatient specialist health services and to the definition of the essential level of assistance founded by the Italian national healthcare system. In addition, this nomenclature was produced in 1999 35 and it was excluded from the rapid evolution of the clinical care world. These limits led to the creation of many different local terminologies which represent an obstacle to achieving information interoperability.

For these reasons, a HTS, whose interface is compliant to the CTS2 standard, was also included in the architecture design in order to permit the sharing of information semantics. The Health Terminology Service provides functionalities to search and query structured terminological content pertaining to code systems and therefore allows the mapping of a code of a specific code system in the corresponding code of the reference code system. In this work, the adopted reference code system is the standardized vocabulary LOINC (Logical Observation Identifiers Names and Codes) 36. Thanks to this service, the HRMS is able to modify all the codes of a specific code system in the corresponding LOINC code to allow the transmitted CDA R2 to be correctly interpreted by both external clinician/research and computer processes.

The other class of CDA R2 elements, which the HRMS must manage, is related to the patient identifiers (ID). In fact, a person in his/her lifetime may have episodes of care provided by several healthcare organizations, many of whom assign and maintain the patient's identifier autonomously. In this context, each organization or even department often assigns its own ID, which uniquely iden-

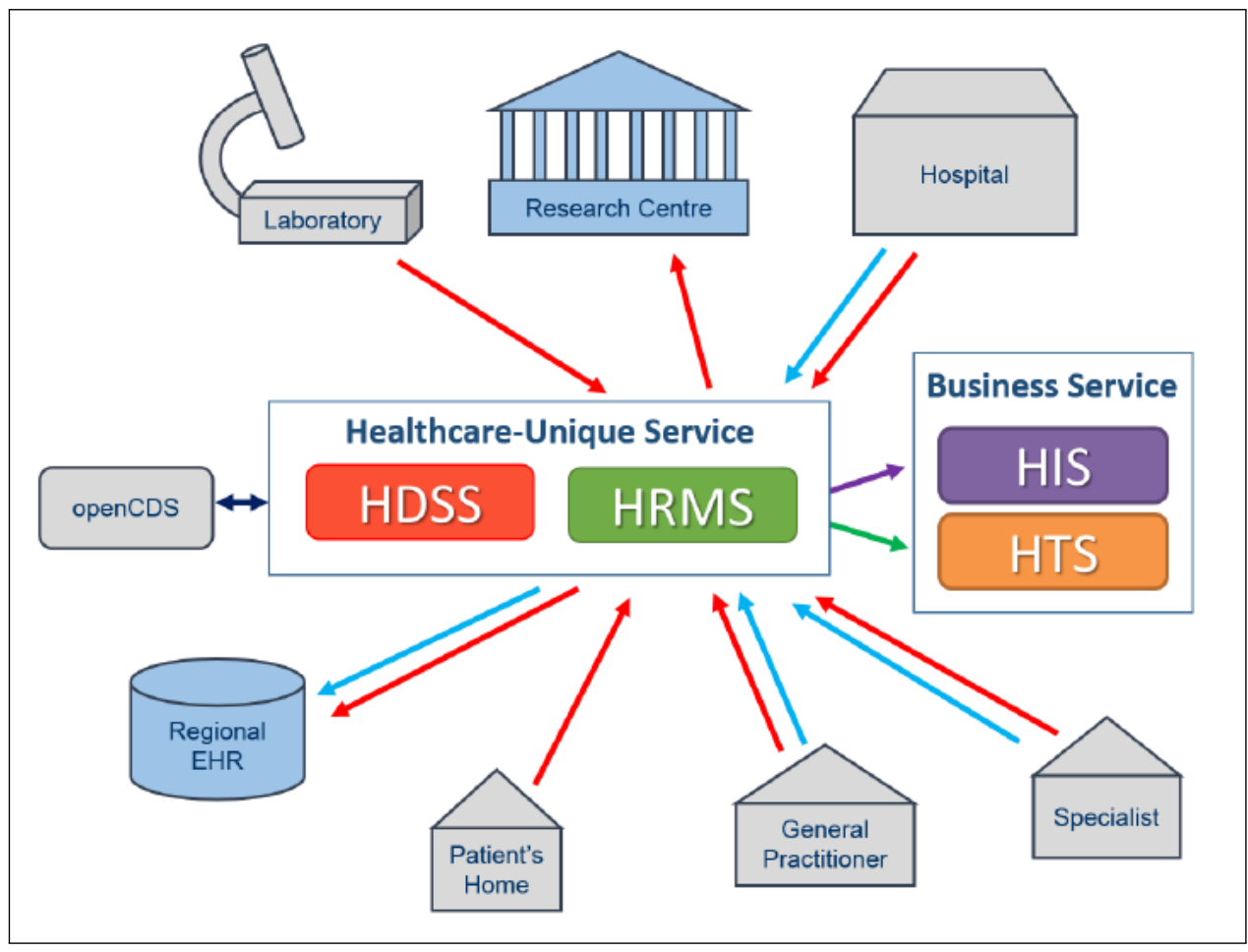

Figure 1: The proposed Service Oriented Architecture compliant to HSSP specifications. Grey actors represents systems that interacts with the central services through client applications, while light blue ones represents actors with provide access to the content of their system with RLUS web service interfaces. Red arrows represent calls to RLUS Put operation, light blue arrows reppresents calls to RLUS Get operations, purple and green arrows respectly represents call to IXS and CTS2 query operations and blue arrows represents interactions with external decision support systems.. 
tifies the patient for its own purposes, with the result that these ID values are meaningless outside that system or organization. In order to manage all the identifiers, the authors also introduced a HIS, whose interface is compliant to the IXS standard. The Health Identity Service provides query operations, given an identifier, to retrieve the list of all other IDs, which are linked to it. In this solution, as a reference identifier the Italian fiscal code provided by the Italian Economy and Finances Ministry was adopted. The reason for this choice is that the same code is used by the Italian Health Ministry to identify patients within the Italian National Health System. By calling these functionalities, the HRMS can modify all identifiers assigned by a specific system (root) in the corresponding ID by referring to the root of the address system. The use of the Italian fiscal code is limited to the cases in which the identity of the patient is indispensable; in all other cases, the privacy of the patients is maintained by the automatic work of the HIS. After these changes, the CDA R2 is ready to be addressed to the specific actor, typically the regional EHR or the center responsible for research activity in which the patient is involved.

The other class of functionality provided by the HRMS is the get operations, which are used to access a patient's information; a classic case is when a general practitioner, a specialist, or a department/care unit wish to be informed about the patient's clinical history stored within the EHR. When the HRMS receives a request to get a resource related to a specific patient, it first queries the HIS to obtain the corresponding patient's identifier within the regional EHR, where the information is stored. If the applicant is authorized to have access to the clinical data, then the Health Record Management Service interacts with the EHR to obtain the resource. Before sending the CDA R2 request, the HRMS queries the HTS to verify if for each code referring to LOINC, there is a corresponding mapped code in the local code system defined for the specific system which is requesting the information. In the case in which a corresponding mapped code is found, the HMRS integrates the LOINC code with the local code obtained and then addresses the standardized document to the applicant.

The last service, which the authors included in the described architecture, is the HDSS, whose interface is compliant to the CDSS standard. This service allows the EHR to interact with the international medical community, in order to improve the relevant shared data, which can be processed to provide patient-specific assessments or recommendations. In this case, the information is mapped using vMR, as indicated by CDSS standard. Finally, to set up this architecture, dedicated interfaces and clients were also designed in addition to the standardized services mentioned in order to allow each actor to communicate with the services in a standardized manner.

\subsection{Status of the implementation}

The first service that the authors designed and developed was the HRMS. A Windows Communication Foundation (WCF) Service 37, whose interface is compliant with RLUS standards and are described through Web Service Description Language (WSDL) files, was implemented. One of the advantages of the RLUS is its flexibility and adaptability to different semantic content. These concepts are realized by the separation of functionality and semantic content in the interfaces. Relevant semantic content is designated by a Semantic Signifier that is defined for client use through XSD (XML Schema Definition) files. The principal Semantic Signifier used to allow the communication between actors involved in this architecture was the CDA R2.

Then the authors considered HTS. For the design, they started to implement a terminology repository in order to store all the information needed to manage clinical and health codifications and terminologies. In this phase, the specifications provided by OMG in the CTS2 STM were adopted. In detail, CodeSystem, CodeSystemVersion, EntityDescription, MapCatalog, MapVersion, MapEntry resources of CTS2 STM were considered. In the same time, the authors started the implementation of the interfaces of the HTS. For each CTS2 resource, the authors planed the development of WCF services to support read, query, maintenance and history functionality categories. At the present, the terminology repository is ready to manage all these capabilities for each CTS2 resource type, while services to provide read and query functionalities for CodeSystem are available.

Lastly, the HIS was designed. The authors implemented a repository to manage entities and implemented WCF services to manage patient's identifiers. Therefore, Patient class of CDA R2 was chosen as semantic signifier.

The proposed architecture and these implemented WCF service have already been adopted in different systems. The first one was used within the Artemis funded Project CHIRON [38. CHIRON is an acronym for "Cyclic and person-centric Health management: Integrated appRoach for hOme, mobile and clinical eNvironments". It intends to propose an integrated framework designed to allow a person-centric health management throughout the complete care cycle, focused on patients affected by Congestive Heart Failure (CHF). Within the CHIRON telemonitoring system, the described Health Record Management Service was used to allow the standardized communication between the monitoring platform [39] located in the patient's home and the institutional openEHR based EHR, used as the core of the internal CDS system 40. The authors collaborated on the development of the client hosted in a patient's home which provides clinical data to the HMRS through CDA R2 and implemented the EHR interface, which receives the tele-monitored data and converts the information mapped in CDA R2 in information mapped using the openEHR approach [41. 
A second system which the authors implemented was a solution to realize the "Interoperable" tier indicated the EHRCR (Electronic Health Records for Clinical Research) Functional Profile Working Group 42 in order to manage clinical trials on HIV patients. The core of this solution was formed by the HRMS and the HIS that automatically orchestrated the bi-directional communication between the hospitals and research centers. The HRMS was responsible for managing clinical data, while the HIS, had the same purpose for administrative data. At present, this system involves two hospitals and four regional and national research centers 43 .

Some client and web servers implemented in this solution were also adopted in another solution to connect a tool to manage both clinical data and clinical trials in ophthalmology and the one of the involved hospitals [44.

Another solution in which the authors are applying this architecture is for the surveillance of antibiotic multiresistant microorganisms; the implementation of all clients and interfaces is being coordinated. These solutions will support the communication between Infectious Diseases Departments, Departments of Neurosciences, Ophthalmology and Genetics, advanced Ligurian and Italian research centers and external CDS systems.

Finally, the HTS will be the focus of a project with the Veneto region. The HTS will be adopted to manage semantics between the ACG (Adjusted Clinical Groups) and the regional EHR.

\section{Conclusions}

In this paper a landscape architecture, supporting the reuse of clinical data and enhancing collaboration between the actors involved in the treatment of illness throughout the complete care cycle, has been described and discussed. The core of this architecture consists of four standardized services, based on the HSSP specifications, which allow the transmission and interpretation of clinical data enclosed within HL7 v3 CDA R2 between hospitals, general practitioners, specialists, laboratories, the regional EHR, clinics or patient's home and advanced research centers. In the present state of implementation, this architecture is realized through:

- A Health Record Management Service compliant to RLUS standards

- A Health Identity Service compliant to IXS standard

- A Heath Terminology Service which provides a subset of functionalities indicated by CTS2 standard

- A set of specific clients that permit the existing software to interface with these services. This implementation was successfully employed within three systems:

- A tele-monitoring system to support the continuity of care of chronic patients affected by $\mathrm{CHF}$
- An architecture to manage clinical trials on HIV patients

- An solution to manage clinical trials in ophthalmology

One of the positive aspects of this proposed landscape architecture is certainly its flexibility which allows the system to be future proof, adding and integrating new functionalities to an existing solution. This feature permits the reuse of software, which was financed by previous investments; a fundamental element to be approved by healthcare organizations. In fact, while services were developed ex novo by the authors, the client applications were developed as an additional tool that interacts with the existing system, adding functionalities. In addition, the applicative solutions were designed in close collaboration with the medical staff in order to satisfy all requirements; a crucial point in order to be accepted by the final users. In fact, S. Fernando et al. declared that the major reason which caused the failure of NPfIT was directly linked to the clinicians' reluctance to accept new IT systems because it was affirmed that the proposed solution provided little positive impact on making their job easier or improving patient care 15 .

The authors' experience, received through the collaboration with clinicians, technicians and patients, teaches that one of the most required features is the transparency to the final user. All actors would only approve a solution if it did not necessitate a serious change in their treatment of illness and would consequently produce an important improvement in patient care or a consistent decrease of human errors or time consumption. For example, the insertion of the Health Decision Support Services within the architecture was prompted by clinicians' request to provide data to external CDS systems.

\section{Acknowledgements}

The research work for the design of this landscape architecture started within CHIRON project of the European Joint Undertaking on Embedded Systems ARTEMIS which was co-funded by the EU and by the National Authorities (CHIRON: GA n. 100228).

The authors would like to thank: the CHIRON partners (in particular Tullio Salmon Cinotti, Fabio Vergari, Francesco Morandi, Jan-Marc Verlinden, Kevin Keene and Carlos Cavero Barca for the collaboration in research and implementation activities and Silvio Bonfiglio and Letizia Gabrielli for the coordination activities); Luciano Apollaro, Monica Bonetto, Paolo Fraccaro, Barbara Giannini, Matteo Macr?, Emilio Masi, Maria Eugenia Monteverde, Laura Pastorino, Valeria Pupella, Matteo Visciola for the collaboration in software implementation; Marco Anselmo, Giovanni Cassola, Giovanni Cenderello, Pasqualina De Leo, Chiara Dentone, Antonio Di Biagio, Daniela Fenoglio, Massimo Gazzarata, Giovanni Mazzarello, Massimo Nicol?, Maurizio Setti, Claudio Viscoli for the medical collaboration and support. 


\section{References}

[1] I. Iakovidis, "Towards personal health record: current situation, obstacles and trends in implementation of electronic healthcare record in Europe," Int. J. Med. Inform., vol. 52, no. 1 , pp. 105-115, 1998.

[2] M. della Salute, "Il Fascicolo Sanitario Elettronico. Linee Guida Nazionali," Sole 24Ore Sanit?, vol. 7, 2011.

[3] A. Ströher and W. Honekamp, "ELGA-die elektronische Gesundheitsakte vor dem Hintergrund von Datenschutz und Datensicherheit," Wiener Medizinische Wochenschrift, vol. 161, no. 13-14, pp. 341-346, 2011.

[4] R. Krippes, M. Schwebag, and S. Benzschawel, "Country Brief: Luxembourg," 2010.

[5] Center for Quality Region Southern Denmark, "Report: Systematisk journalgennemgang med IHI's GLOBAL TRIGGER TOOL. Erfaringer og resultater fra afpr?vning p? danske sygehuse."

[6] "The National Patient Safety Campaign of Norway In Safe Hands."

[7] HIMMS, "Electronic Health Records: A Global Perspective $2008, " 2008$

[8] A. D. Black, J. Car, C. Pagliari, C. Anandan, K. Cresswell, T. Bokun, B. McKinstry, R. Procter, A. Majeed, and A. Sheikh, "The impact of eHealth on the quality and safety of health care: a systematic overview," PLoS Med., vol. 8, no. 1, p. e1000387, 2011.

[9] L. Catwell and A. Sheikh, "Evaluating eHealth interventions: the need for continuous systemic evaluation," PLoS Med., vol. 6, no. 8, p. e1000126, 2009

[10] L. D. Schaeffer, A. M. Schultz, and J. A. Salerno, HHS in the 21st Century:: Charting a New Course for a Healthier America. National Academies Press, 2009.

[11] C. P. Waegemann, "Current Status of EPR Developments in the US," Towar. an Electron. Heal. Rec., vol. 99, pp. 116-118, 1999.

[12] F. Ueckert, M. Goerz, M. Ataian, S. Tessmann, and H.-U. Prokosch, "Empowerment of patients and communication with health care professionals through an electronic health record," Int. J. Med. Inform., vol. 70, no. 2, pp. 99-108, 2003.

[13] F. Thiessard, F. Mougin, G. Diallo, V. Jouhet, S. Cossin, N. Garcelon, B. Campillo-Gimenez, W. Jouini, J. Grosjean, and P. Massari, "RAVEL: retrieval and visualization in ELectronic health records.," in MIE, 2012, pp. 194-198.

[14] J. Wyatt, "Medical informatics, artefacts or science?," Methods Inf. Med., vol. 35, no. 3, pp. 197-200, 1996.

[15] S. Fernando, J. Choudrie, M. Lycett, and S. de Cesare, "Hidden assumptions and their influence on clinicians' acceptance of new IT systems in the NHS," Inf. Syst. Front., vol. 14, no. 2, pp. 279-299, 2012.

[16] HSSP, HL7, and OMG, "The Practical Guide for SOA in Health Care - A real-world approach to planning, designing, and deploying SOA, Version 1," 2008.

[17] "Open Clinical Decision Support (openCDS)." [Online]. Available: http://www.opencds.org/

[18] R. H. Dolin, L. Alschuler, S. Boyer, C. Beebe, F. M. Behlen, P. V Biron, and A. S. Shvo, "HL7 clinical document architecture, release 2," J. Am. Med. Informatics Assoc., vol. 13, no. 1, pp. 30-39, 2006.
[19] K. Kawamoto, C. Nanjo, D. Shields, V. Lee, A. Boxwala, M. Roche, B. Rhodes, D. Sottara, M. AK, Y. Bao, H. Strasberg, P. Tattam, S. Bolte, P. Scott, K. Boone, Z. Liu, C. Melo, N. Hulse, J. Basilakis, R. Worden, D. Chertcoff, C. Curtis, G. Del Fiol, E. Fry, J. Dufour, and L. Charlois, "HL7 Version 3 Domain Analysis Model: Virtual Medical Record for Clinical Decision Support (vMR-CDS), Release 2," 2013.

[20] L. Alschuler, C. Beebe, K. W. Boone, R. H. Dolin, B. Marquard, L. Bryan, R. Grapp, M. Morsch, and S. Hardy, "Implementation Guide for CDA Release 2.0 Care Record Summary Release 2 Discharge Summary, Draft Standard for Trial Use," 2009 .

[21] R. H. Dolin, L. Alschuler, C. Beebe, F. M. Behlen, K. W. Boone, D. Gabriel, R. Geimer, G. Giannone, V. "Juggy" Jagannathan, L. McKnight, P. Mitchell, R. Peters, D. Russler, A. Shabo, S. E. Waldren, P. A. Van Dyke, B. Yencha, and A. Zuckerman, "HL7 Implementation Guide: CDA Release 2 Continuity of Care Document (CCD)," 2007.

[22] "HL7 Italia, Implementation Guide Clinical Document Architecture (CDA) Rel. 2 Rapporto di Medicina di Laboratorio," 2009

[23] "IHE Laboratory Technical Framework, vol. 3 (LAB TF-3) Content, rev. 2.1 - Final Text," 2008.

[24] G. Gazzarata, R. Gazzarata, and M. Giacomini, "A Standardized SOA Based Solution to Guarantee the Secure Access to EHR," Procedia Comput. Sci., vol. 64, pp. 1124-1129, 2015.

[25] K. Kawamoto, A. Honey, and K. Rubin, "The HL7OMG Healthcare Services Specification Project: motivation, methodology, and deliverables for enabling a semantically interoperable service-oriented architecture for healthcare," J. Am. Med. Informatics Assoc., vol. 16, no. 6, pp. 874-881, 2009

[26] "HL7 Version 3 Standard: Retrieve, Locate, and Update Service (RLUS) Release 1." [Online]. Available: http://www.hl7.org/implement/standards/product_brief. cfm?product_id $=89$

[27] S. Lotti, J. Koisch, A. P. Honey, S. Robinson, and K. Kawamoto, "Service Functional Model Specification Retrieve, Locate and Update Service (RLUS), Release 1," 2012.

[28] "Software Engineering Institute (SEI) Software Architecture Glossary." [Online]. Available: http://www.sei.cmu.edu/ architecture/start/glossary/.

[29] R. Hamm, A. Estelrich, N. Canu, F. Oemig, and S. Nachimuthu, "HL7 Common Terminology Services, Release 2: Service Functional Model Specification, Normative Release," 2013.

[30] OMG, "Retrieve, Locate, and Update Service (RLUS) Specification, Version 1.0.1," 2011.

[31] G. Del Fiol, R. Jenders, K. Kawamoto, H. Strasberg, O. Young, D. Boaz, D. Shields, B. Esler, K. Rubin, M. Koethe, A. Kirnak, C. Curtis, R. Hausam, and A. Honey, "HL7 Version 3 Standard: Decision Support Service (DSS), Release 1," 2011.

[32] OMG, "Common Terminology Services 2, Version 1.2," 2015. [Online]. Available: http://www.omg.org/spec/CTS2/1.2/

[33] P. Gilbert, A. Honey, A. Kirnak, S. Lotti, D. Ries, and G. Teichrow, "HL7 Version 3 Standard: Identification and CrossReference Service (IXS) Release 1," 2013.

[34] OMG, "Identity Cross-Reference Service (IXS), Version 1.0.1," 2011.

[35] Italian Decree of 27 August 1999, Gazzetta Ufficiale of 27 September 1999 n. 227. 
[36] "Logical Observation Identifiers Names and Codes (LOINC)." [Online]. Available: http://loinc.org/

[37] W. Zhang and G. Cheng, "A service-oriented distributed framework-WCF," in Web Information Systems and Mining, 2009. WISM 2009. International Conference on, 2009, pp. 302-305.

[38] "CHIRON Project." [Online]. Available: https: //artemis-ia.eu/project/17-chiron.html

[39] F. Vergari, T. S. Cinotti, A. D'Elia, L. Roffia, G. Zamagni, and C. Lamberti, "An integrated framework to achieve interoperability in person-centric health management," Int. J. Telemed. Appl., vol. 2011, p. 5, 2011.

[40] M. Luštrek, B. Cvetković, M. Bordone, E. Soudah, C. Cavero, J. M. Rodríguez, A. Moreno, A. Brasaola, and P. E. Puddu, "SUPPORTING CLINICAL PROFESSIONALS
IN DECISION-MAKING FOR PATIENTS WITH CHRONIC DISEASES."

[41] R. Gazzarata, F. Vergari, T. Salmon Cinotti, and M. Giacomini, "A standardized SOA for clinical data interchange in a cardiac telemonitoring environment," IEEE J Biomed Heal. Inf., vol. 18 , no. 6 , pp. $1764-1774,2014$

[42] EHR/CR Functional Profile Working Group, "EHR/CR User Requirements Document," 2008.

[43] R. Gazzarata, B. Giannini, and M. Giacomini, "A SOA-based platform to support clinical data sharing," Informatics Heal. Soc. Care.

[44] M. Bonetto, M. Nicolo, R. Gazzarata, P. Fraccaro, R. Rosa, D. Musetti, M. Musolino, C. E. Traverso, and M. Giacomini, "I-Maculaweb: A Tool to Support Data Reuse in Ophthalmology," IEEE J. Transl. Eng. Heal. Med., vol. 4, pp. 1-10, 2016. 